\title{
Spatial analysis linking landscape features and genetic population structure in cougars (Puma concolor) in the northern Rocky Mountains
}

\author{
David C. Wheeler and Lance A. WalleR*†
}

Landscape genetics is a field of research that seeks to understand the drivers of the observed spatial distribution of genetic variation in a species of plant or animal using methods from population genetics, landscape ecology, geography, and spatial statistics. One of the important research areas in landscape genetics is to identify landscape barriers to genetic flow. Barriers can limit interaction of organisms and hence lead to genetic structure (i.e. frequency of genotypes) in a population that becomes increasingly spatially structured over time. Here, we investigate model-based spatial methods to assess the relationship between landscape and genotype distributions of cougars (Puma concolor) in Western North America. Previous research has assessed genetic differentiation in cougars in North America using a non-spatial Bayesian clustering model and found evidence of genetic population structure in cougars, with suggestive but indistinct spatial boundaries between subpopulations. To determine if including spatial information on samples in a classification model would refine the observed spatial signal within the genetic population structure, we applied Bayesian classification models to microsatellite loci data with associated spatial locations. The spatial model revealed two clearly differentiated cougar subpopulations, in contrast to the two overlapping subpopulations suggested by methods not accounting for space. We also explored through geographic information systems and generalized linear models whether resulting genetic population structure boundaries aligned with landscape features. The spatial correspondence of genetic subpopulations and a major river and road is suggestive of possible landscape barriers to cougar movement. This study demonstrates that the use of explicit spatial information and Bayesian classification models adds novel insight when investigating genetic population structure in a species.

AMS 2000 SUBJECT ClASSifiCATIONS: Primary 62P07; secondary 92B08.

\footnotetext{
${ }^{*}$ Corresponding author.

†This research is supported in part by NIEHS (NIH) grant R01 ES015525 (LAW). The views presented herein represent those of the author and do not necessarily represent those of NIEHS or NIH.
}

KeYwords AND PHRASEs: Bayesian, Classification, Landscape genetics, Microsatellite, Geneland.

\section{INTRODUCTION}

Landscape genetics is a field of research seeking to understand the spatial distribution of genetic variation in species of plants or animals using methods and data from population genetics, landscape ecology, geography, and spatial statistics (Storfer et al. 2010). The main distinction between traditional population genetics and the more recently developed field of landscape genetics is the integration of tests of landscape heterogeneity on gene flow and the genetic variation between and within populations (Holderegger and Wagner 2008). In a recent survey of the landscape genetics literature, Storfer et al. (2010) found that while interest in using landscape genetic approaches to understand how genetic variation is affected by landscape processes has grown markedly in recent years, very few analyses make use of spatial analytic methods to address what is, at its heart, a spatial problem. A few recent works describe the (limited) use of spatial statistics within landscape genetics (Storfer et al. 2007; Guillot et al. 2009). The continuing improvements in technology to generate molecular data and the increasing availability of high-resolution spatial data ensure that the number of landscape genetics studies will continue to increase (Storfer et al. 2010). The growing need for more explicitly spatial methods in the field of landscape genetics should leave spatial statisticians poised to make significant contributions to the field.

One recurring research question in landscape genetics is how to accurately identify and quantify barriers to genetic flow. Landscape barriers can limit interaction of organisms and therefore lead to spatially explicit genetic structure (i.e. frequency of genotypes) in a population. The idea of such structure among genetic subpopulations has been the subject of work in population genetics (Hartl 2000; Hedrick 2004; Rousset 2004), ecological genetics (Conner and Hartl 2004), and phylogeography (Avise 2000). Early work by Wright $(1943 ; 1946)$ linked genetic structure to spatial distance between populations. Recent efforts attempt to expand empirical methods to use spatial pattern to describe 
population genetics (Epperson 2003; Guillot et al. 2009). Understanding the role that landscape has on species differentiation is particularly important for conservation ecology, where the preservation of particular habitats is of interest (Allendorf and Luikart 2006; Frankham et al. 2010). Identification of barriers to gene flow can also assist in the prediction of the spatial spread of disease within a host population and can be used for disease management (Storfer et al. 2010). For example, in the case of rabies in raccoon populations, the knowledge that rivers act as barriers has been useful for targeting vaccinated baits as a control measure in areas where landscape features have facilitated gene flow (Real and Biek 2007).

There are many examples in the ecology literature of researchers investigating genetic population structure in animals or plant species that align with landscape barriers or habitat divisions, both natural and anthropogenic. Guillot et al. (2009) provide a recent review of some of this literature. Coulon et al. (2006) found that two genetically differentiated subpopulations of roe deer in southwestern France aligned with a linear area containing several barriers, including a highway, a river, and several canals. Frantz et al. (2006) identified the Moselle river valley as a barrier for dispersal of red deer in Europe. Riley et al. (2006) found that genetic population structure in bobcats and coyotes corresponded to a major roadway. Keyghobadi et al. (1999) discovered that forests were barriers to gene flow for alpine butterflies in Canada. Castric et al. (2001) found that waterfalls were associated with more genetic isolation in brook charr in Maine. Funk et al. (2005) determined that mountain ridges and elevation differences were associated with genetic differentiation in Columbia spotted frogs in western Montana and Idaho. Epps et al. (2005) discovered that genetic diversity in desert bighorn sheep in California was limited by the anthropogenic barriers of highways, canals, and urban areas. Sacks et al. $(2004 ; 2008)$ found genetic structure in coyotes in California associated with habitat divisions. Piertney et al. (1998) uncovered evidence suggesting that unsuitable habitat along a river inhibited gene flow between populations of red grouse in Scotland and Cegelski et al. (2003) suggested that limited gene flow in Montana wolverines was related to human development. Storfer et al. (2010) also provide an extensive summary of these and other studies that have focused on understanding landscape barriers to gene flow. While linear features, such as roads, rivers, and mountain ranges have been found to be barriers to gene flow, the effects of putative barriers on gene flow also appear to vary by species.

Our work seeks to identify landscape barriers to gene flow by assessing spatial genetic population structure in a sample of cougars (Puma concolor) in North America. The cougar is a large predatory feline distributed throughout the Americas. Cougars are solitary predators that usually avoid human contact and prefer to hunt by stalking behind vegetation and land cover (Cougar Discussion Group 2009). Home ranges for male cougars have been estimated to be between 25 and 380 square miles (Cougar Discussion Group 2009). Cougar movement is thought to be influenced by both natural and manmade landscape barriers, which over time could lead to spatially structured genetic population structure. Previous studies have investigated genetic population structure in cougars in various areas of North America (Sinclair et al. 2001; Ernest et al. 2003; Anderson et al. 2004; McRae et al. 2005; Biek et al. 2006). This present study builds upon previous research of a sample of cougars in the northern Rocky Mountain region of North America by Biek et al. (2006). The northern Rocky Mountains experienced rapid declines in cougar populations during the early 20th century due to human persecution and depleted prey populations, but populations have rebounded following legislation limiting the take by hunters of cougars and their ungulate prey (Biek et al. 2006). In an analysis of genetic population structure in this sample of cougars, Biek et al. (2006) identified two cougar subpopulations based on genetic data using a (non-spatial) Bayesian clustering model in the software program structure (Pritchard et al. 2000). While genetically distinct, the two subpopulations exhibit considerable spatial overlap when mapped. A possible explanation for the indistinct boundary observed between subpopulations is that there was no consideration of spatial correlation of cougar genetic data in the model and, if spatial autocorrelation is present, this omission could reduce the power of the approach to clearly identify genetic differentiation that may be caused by the effect of local landscape barriers on host movement. The latent structure in the results of Biek et al. (2006) raises the question whether the spatial locations of individual cougars can be used to refine inference on possible links between subpopulation membership and location.

To investigate the hypothesis that gene flow in the North American cougar population (analyzed by Biek et al. 2006) is affected across space by the landscape, we used spatial Bayesian clustering models implemented in the software Geneland (Guillot 2008; Guillot et al. 2008) to determine the number and composition of genetically distinguishable cougar subpopulations. These models extend the basic structure model to explicitly account for the spatial location of sampled observations and include a priori spatial correlation in the genetic data. The subpopulation composition provided by the models includes the posterior probabilities of subpopulation membership for each individual. We then quantified associations between modeled genetic population structure and certain relevant landscape features using generalized linear models (GLMs) and generalized additive models (GAMs) and landscape data accessed via a geographic information system (GIS).

\section{MATERIALS AND METHODS}

\subsection{Data}

Our data contain information for 273 cougars caught for research purposes or killed by hunters in the northern Rocky 
Mountain regions of Montana and Idaho and extending into Canada during 1990-2004. The dataset included geographic coordinates for the sample location of each cougar, or the hunting area centroid for some hunter-killed cougars. The dataset also contained genetic information in the form of microsatellite data containing 11 loci where variation is expressed in cougars. Microsatellites are repeating sequences of base pairs of DNA used as molecular markers in genetics, and a locus is the specific location of a gene or DNA sequence on a chromosome. Ideally, for each cougar there is an allele pair for each of the 11 loci, although some cougars were missing allele data, where an allele denotes the particular form of the DNA sequence of a specific gene. The genotype for each cougar then consists of 11 loci with two alleles per locus. Each observed allele is the result of the assumed process of random mating. Specifically, the 11 microsatellite loci under analysis include Fca30, Fca35, Fca57, Fca77, Fca90, Fca96, Fca132, Fca176, Fca391, Fca559, and Lc109 (see Biek et al. 2006 for more information). The genetic data came from previous lab analysis of DNA from either blood or tissue samples taken from the salivary glands or lymph nodes using standard techniques and protocols (e.g. polymerase chain reaction (PCR)). The following loci had null alleles due to problems with PCR amplification for a number (n) of subjects: Fca30 (4), Fca57 (2), Fca90 (7), Fca96 (1), Fca132 (2), Fca176 (2), Fca559 (3). All cougar genetic data used in this project were previously analyzed (aspatially) by Biek et al. (2006).

\subsection{Bayesian clustering models for genetic data}

We used Bayesian classification models for microsatellite loci data to investigate genetic population structure. Bayesian clustering models have become a popular tool in population genetics to detect and quantify the factors affecting genetic structure and gene flow (see, for example, Frantz et al. 2009; McCairns and Bernatchez 2008). The Bayesian clustering models used in population genetics are more appropriate for quantifying genetic structure than the traditional clustering methods found in the data mining and unsupervised learning literature (see Hastie et al. 2001; Berk 2008) because they are designed for genetic data that are categorical and multivariate, as a genotype is defined by multiple loci (with potentially many alleles). In addition, these Bayesian clustering models can account for the complexities of spatial correlation in genotypes and correlation in allele frequencies when determining genetic population structure. Moreover, the Bayesian framework is quite flexible and provides a convenient approach to model a variety of potential structures in genetic data.

Several of these Bayesian classification models for genetic data have been implemented in the widely-used software packages structure and Geneland. There are similarities in the model in structure and the models available in Geneland, but the main important difference is in the assumption or not of spatial correlation in genotypic data taken from different geographic locations. The model in structure assumes population membership is defined by a set of exchangeable (conditionally independent given hyperparameter values) random intercepts implemented through i.i.d. prior distributions on population membership. In fact, the model in structure does not include spatial information associated with genotypes in the model at all; it is an aspatial model. From the perspective of exploring landscape barriers that may affect genetic flow this is a disadvantage, as any spatial signal in the data may be obscured. Therefore, the aspatial model found in structure is of interest here only for comparative purposes (with earlier research by Biek et al. 2006). Instead, we focus our attention on the spatial Bayesian model in Geneland when exploring genetic population structure of cougars.

The key assumption in the spatial model in Geneland is that genotypes are spatially correlated, i.e. that proximate observations are more similar than distant ones. This assumption of spatially correlated genotypes is useful for exploring possible spatial patterns that may arise when population differentiation occurs by limited gene flow influenced by the occurrence of landscape barriers, such as major rivers, major roads, mountain ranges, and human activity. The assumption allows spatial breaks in genotypic data due to spatial features to be expressed in population subgroups more readily than in a model with an assumption of independence in population membership. The model naturally incorporates the spatial locations of sampled organisms for its assumption of spatial correlation.

The spatial Bayesian classification models in Geneland are detailed in Guillot et al. (2005a; 2005b) and we provide a brief overview of key model details here. There are several different models in Geneland, with options for modeling allele frequencies that are either correlated or uncorrelated across populations (not to be confused with spatial dependence in genotypes); null alleles; and uncertainly in sampling locations. The Bayesian clustering models available in Geneland, and also in structure, assume that for each subpopulation, allele frequencies are approximately at Hardy-Weinberg (HW) equilibrium, and that there is linkage equilibrium (LE) between loci across subpopulations. Hardy-Weinberg equilibrium refers to situations where several conditions are met: mating is random within populations, population size is large, migration and mutation effects are insignificant, alleles studied are not under selection, and the alleles separate by Mendelian inheritance (Freeland 2006). Linkage equilibrium occurs when the association between alleles both within each locus and between loci is random; alleles will not be more likely to occur together than expected based on their separate frequencies in the population with LE (Freeland 2006). Linkage disequilibrium occurs when there is non-random association between alleles at different loci. 
The data are defined as $\mathbf{s}=\left(s_{1}, \ldots, s_{n}\right)$ for the $n$ sampling locations, where $s_{i}$ is a two-dimensional vector of coordinates $\left(s_{i 1}, s_{i 2}\right)$, and the genotypes for $n$ cougars $\mathbf{z}=$ $\left(z_{1}, \ldots, z_{n}\right)$, where $z_{i}$ is a vector of $L=11$ allele pairs (for diploid organisms) for each of $l=1, \ldots, L$ loci and with individual pairs denoted $z_{i, l}=\left\{\alpha_{i, l}, \beta_{i, l}\right\}$. The genotypes $\mathbf{z}$ comprise $K$ subpopulations, where $K$ is either fixed or estimated in the model. When $K$ is estimated, it is a priori assumed to follow a uniform distribution, $K \sim \operatorname{Uniform}\left(K_{\min }, K_{\max }\right)$, between a user-specified minimum and maximum. The allele frequency of allele $j$ in locus $l$ in subpopulation $k$ is denoted $f_{k l j}$. Allele frequencies in the subpopulations being sought are unknown. For each subpopulation $k$ and locus $l$, the allele frequencies in the vector $f_{k l 1}, \ldots, f_{k l J_{l}}$ sum to one, where $J_{l}$ is the number of alleles per locus. The allele frequencies are considered multinomial probabilities and a conjugate prior probability distribution, with the unit summation condition, is the Dirichlet distribution (Guillot et al. 2005a).

The allele frequencies may be assumed to be either uncorrelated or correlated across subpopulations, yielding two types of models in Geneland. In the simpler uncorrelated allele frequency model, the allele frequencies are assumed to follow independent Dirichlet prior distributions, $f_{k l}$. $\operatorname{Dirichlet}(\gamma, \ldots, \gamma)$. The uncorrelated allele frequency model, however, does not account for the situation where some allele frequencies are correlated across subpopulations. The correlated allele frequency model adds correlation between allele frequencies from different subpopulations by introducing a theoretical ancestral population from which current subpopulations have drifted with drift factors $d_{k}$, which quantify the level of genetic differentiation for each subpopulation from the ancestral population and may be interpreted as $F_{S T}$ values (Guillot 2008), a commonly used measure in genetics for population differentiation. The allele frequencies for each locus from the ancestral population are denoted $f_{A l j}$ and assumed to follow a Dirichlet distribution, $f_{A l}$. Dirichlet $(1, \ldots, 1)$. The allele frequencies in the current subpopulations are $f_{k l} . \sim \operatorname{Dirichlet}\left(f_{A l 1} \frac{1-d_{k}}{d_{k}}, \ldots, f_{A l J_{l}} \frac{1-d_{k}}{d_{k}}\right)$, and are conditionally independent across subpopulations given $f_{A}$ and $d$. Given the partitioning of the observations to subpopulations and the allele frequencies $f_{k j l}$, the genotypes in each subpopulation are assumed to be independent draws from the discrete multivariate distribution specified by the $f_{k j l}$, which is equivalent to the assumption of $\mathrm{HW}$ equilibrium within loci and linkage equilibrium between loci (Guillot et al. 2005a).

As previously mentioned, the primary distinguishing factor between the spatial model and the non-spatial model in Geneland is the assumption of spatial correlation of genotypes in the spatial model. This assumption is implemented through a marked Poisson-Voronoi tessellation process which yields a spatially correlated prior structure for the individual population membership $\left(p_{i}\right.$ 's) as follows.
The spatial model assumes that the $K$ subpopulations partition the study area, each occupying non-overlapping areas $\Delta_{1}, \ldots, \Delta_{K}$. In the Poisson-Voronoi tessellation, there are $m$ Voronoi polygons that are aggregated to form the $K$ subpopulations. The polygons have nuclei $\left(u_{1}, \ldots, u_{m}\right)$ that are random and uniformly distributed, $\left(u_{1}, \ldots, u_{m}\right) \stackrel{i i d}{\sim}$ Uniform $(D)$. The number of polygons follows a Poisson distribution, $m \sim \operatorname{Poisson}(\lambda)$. The "mark" $\left(c_{1}, \ldots, c_{m}\right)$ for each polygon defines subpopulation membership (defining the $p_{i}$ 's) and is sampled from a uniform distribution, $\left(c_{1}, \ldots, c_{m}\right) \stackrel{i i d}{\sim}$ Uniform $(1, \ldots, K)$. The amount of spatial organization in the process is controlled by the parameter $\lambda$, which has a uniform distribution with its maximum taken as the number of sampled individuals, $\lambda \sim \operatorname{Uniform}(0, n)$. A small $\lambda$ (and hence $m$ ) results in strong spatial organization, and clearer spatial boundaries between groups. With a very large $\lambda$, each Voronoi polygon would contain at most one observation and the tessellation model would behave similar to an i.i.d. mixture model. Such a mixture model is similar to the prior used in the non-spatial model in structure. In the non-spatial model in Geneland, the prior for the vector of individual subpopulation membership, $\mathbf{p}=\left(c_{1}, \ldots, c_{n}\right)$, is an i.i.d. prior, $\pi(\mathbf{p} \mid K)=1 / K^{n}$. This non-spatial model with the assumption of uncorrelated allele frequencies is the same as the model in structure with an additional assumption of no admixture (Guillot et al. 2005a; Guillot et al. 2005b), where admixture occurs with interbreeding between multiple populations of a species that were previously isolated geographically.

Regardless of the assumption used for correlation of the allele frequencies, the likelihood of the data can be written as

$$
\pi(\mathbf{s}, \mathbf{z} \mid \theta)=\pi(\mathbf{s} \mid \theta) \pi(\mathbf{z} \mid \mathbf{s}, \theta)=\pi(\mathbf{s} \mid \theta) \prod_{i=1}^{n} \prod_{l=1}^{L} \pi\left(z_{i, l} \mid \theta\right),
$$

where the terms of the product are given by the allelic frequencies,

$$
\pi\left(z_{i, l}=(\alpha, \beta) \mid \theta\right)= \begin{cases}2 f_{k l \alpha} f_{k l \beta} & \text { if } \alpha \neq \beta \\ f_{k l \alpha}^{2} & \text { if } \alpha=\beta\end{cases}
$$

Inference on the model parameters is made through the posterior distribution $\pi(\theta \mid \mathbf{s}, \mathbf{z})$. Reversible-jump Markov chain Monte Carlo (MCMC) simulation is used to obtain the joint posterior distribution of the parameters. The MCMC algorithm samples from the joint posterior distribution of the parameters, where the parameter vector $\theta$ depends on the model selected; $\theta=\left(K, m, \mathbf{u}, \mathbf{c}, \mathbf{f}, d, \mathbf{f}_{A}\right)$ for the spatial model assuming correlated allele frequencies, and $\mathbf{f}_{A}$ and $d$ are omitted when assuming uncorrelated allele frequencies.

For the genetic population structure analysis, we used the package Geneland in R. For comparison, we used the spatial and non-spatial models in Geneland to determine the number and composition of genetically differentiated cougar subpopulations under the two different modeling assumptions. 
We followed the suggestion of Guillot et al. (2009) and first estimated $K$ assuming uncorrelated allele frequencies with both the spatial and non-spatial models and then estimated the other parameters in models with $K$ fixed at the previously estimated value. There are some null allele values in the data; hence we used the option for estimating null allele frequencies with the uncorrelated frequency model. In sampling from the joint posterior distribution of the parameters, we used (for every model) 500,000 MCMC iterations of the algorithm, thinning every 100th iteration, with a burn-in of 100,000 iterations. In our application, the results of particular interest include the posterior distributions of $m, \mathbf{u}$, and c yielding posterior inference for local subpopulation assignment and the areas associated with each subpopulation.

\subsection{Comparision of genetic subpopulations with landscape features}

After estimating the genetic subpopulations with Geneland, we sought to evaluate the relationship of the subpopulations to particular landscape features to determine plausibility of a landscape barrier effect on genetic flow in this sample of cougars. There are several examples in the literature of landscape barriers found to be associated with cougar genetic diversity. Ernest et al. (2003) found that genetic subdivision in cougars in California was related to several landscape barriers, including water bodies and human developments. Walker et al. (2000) concluded that genetic spatial structure in cougars in Texas was related to habitat contiguity. McRae et al. (2005) discovered genetic structure in cougars in Arizona, Colorado, New Mexico, and Utah that was related to the habitat barriers of open grassland and desert. Loxterman (2001) found increased genetic population structuring in cougars in Idaho where there was agricultural development.

It is not currently possible in the Bayesian clustering models implemented in Geneland to adjust subpopulation membership probabilities for any covariates, such as landscape features. Instead, we first overlaid predicted subpopulation membership from the spatial model on landscape layers showing major roads, major rivers, and average annual precipitation. We selected in advance those landscape variables thought to impact the movement of cougars over space, notably large highways, large rivers, and a proxy for poor (cougar) habitat. We used ArcGIS (ESRI 2005) to map genetic population structure in terms of estimated subpopulations and landscape thematic layers together to assess their spatial alignment. The landscape thematic layers for major rivers and major roads were supplied by ESRI. A thematic layer for average annual precipitation from 1961-1990 came from the Spatial Climate Analysis Service at Oregon State University.

To better quantify the relationship between landscape features and the genetic subpopulations, we used a GAM (Wood 2006) approach to model the probability of belonging to a particular Geneland subpopulation. We considered a binary response variable $Y$ for membership in a particular group with associated $P(Y=1)=p(s, x)$ depending on landscape variables $x$ and the cougar spatial sampling location $s$. We modeled the log odds of being in a particular group generally as

$$
\operatorname{logit}\left[p_{i}\left(\mathbf{x}_{i}, \mathbf{s}_{i} ; \beta, \eta\right]=\beta^{\prime} \mathbf{x}_{i}+Z(\mathbf{s} ; \eta),\right.
$$

where the left-hand side of the equation is the log of the particular group odds for cougar $i, \beta$ is a vector of regression coefficients that may contain an intercept depending on the model specification, $\mathbf{x}_{i}$ is a vector of covariates for the $i$ th observation with the first element being a 1 if an intercept is included in the model, and $\mathbf{s}_{i}$ contains the two spatial coordinates for the $i$ th observation. $Z(\mathbf{s} ; \eta)$ is a smooth function of the locations and includes the parameters $\eta$ needed for the smoothing function. For covariates, we considered the mean annual precipitation in the area of the sampled location; an indicator variable for the relation of the location to a major river relevant in the area; and an indicator variable for the relation of the location to a major highway relevant in the area. The smoothing function models spatial variation not explained by the covariates. We used thin plate regression splines as the basis for the smooth term with the mgcv package in $\mathrm{R}$. The modeling approach is flexible, and without the smooth term this GAM simplifies to a GLM, specifically it simplifies to a logistic regression model in the case of two groups and to a multinomial logit model in the case of more than two groups. In our analysis, we compare models with and without the smooth term.

To explore the validity of the Geneland results, we applied popular landscape genetics tests to the estimated subpopulations. We tested genotypic differentiation in the Geneland subpopulations with a log-likelihood ratio $G$-statistic test (Goudet et al. 1996; Goudet 2005) in the Genepop software. The $G$-statistic tests the null hypothesis that genotypes are drawn from the same distribution in all subpopulations. We tested the amount of gene flow between the Geneland subpopulations with an $F_{S T}$ test (Weir and Cockerham 1984) in Genepop and an $F_{S T}$ test in the SPAGeDi software package (Hardy and Vekemans 2002) with jackknifing over loci to approximately estimate the standard error of the statistic. $F_{S T}$ is a common measure of population differentiation and is a type of kinship coefficient based on allele identity.

\section{RESULTS}

The spatial and non-spatial models with uncorrelated allele frequencies both found two genetically differentiated subpopulations of cougars (Fig. 1). The subpopulation membership assignments, 1 or 2 in the figure, for each cougar are based on the posterior probabilities of population membership. A finding of one genetic population would indicate a lack of genetic differentiation among cougars, and would suggest no barriers to gene flow in the population. Apparent in the mapped subpopulation membership assignments from 


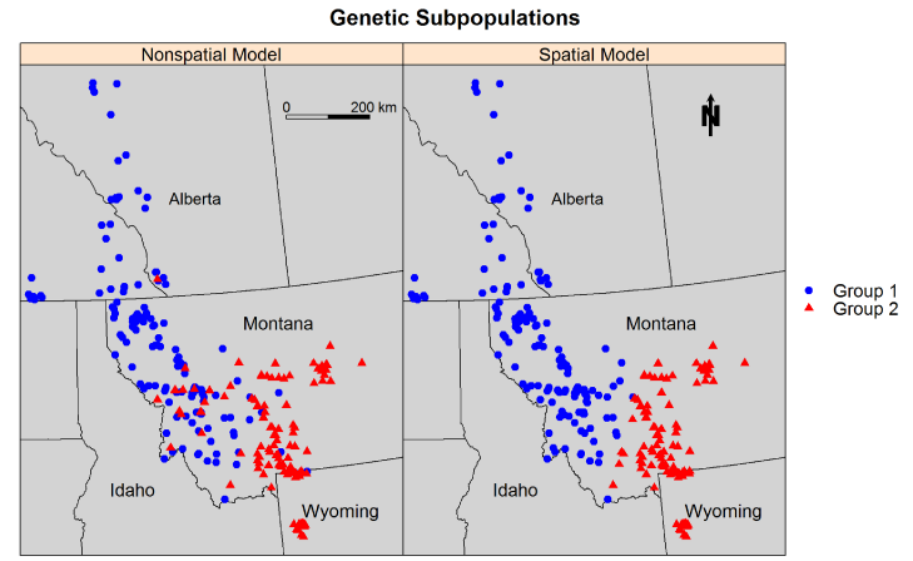

Figure 1. Posterior mode subpopulation assignments from the non-spatial model and spatial model in Geneland for cougars sampled in the study area of the Rocky Mountain region of North America.

the non-spatial model is some spatial overlap in the two subpopulations. There is no clear spatial boundary between the two subpopulations. Rather, the transition between groups appears to occur in the general area of the continental divide, an identifiable landscape feature, with subpopulation 1 generally west of the divide and subpopulation 2 generally east of the divide. In contrast to the non-spatial model, the spatial model finds two spatially distinct genetically differentiated cougar subpopulations. There is effectively no spatial overlap in the subpopulations determined by the spatial model. Viewed this way, the subpopulation membership assignments from the spatial model may be considered a refinement of those from the non-spatial model, where a potentially sharp linear landscape boundary becomes more apparent.

The contrast between the two models in the delineation of cougar subpopulations is more striking when exploring the individual posterior probabilities of subpopulation membership. The contours of posterior probabilities of membership in cougar subpopulation 2 from the spatial model show a steep decline in the probability of belonging to subpopulation 2 (Fig. 2), with a decrease from a probability of 1 to effectively 0 over a very short distance. The spatial model yields stronger spatial organization of the subpopulation areas, effectively separating the study area into two subregions. As a result of reducing the overlap between the subpopulations through increased spatial structure, the boundary between the two subpopulations is more focused and shifted to the east with the spatial model.

In addition to the uncorrelated frequency spatial model fitted above, we also fitted a spatial model with correlated allele frequencies in Geneland and a spatial model with uncorrelated allele frequencies, but with uncertainty in the spatial coordinates for each cougar. The uncertainty is included in the model by treating each true coordinate $t_{i}$ as a sum

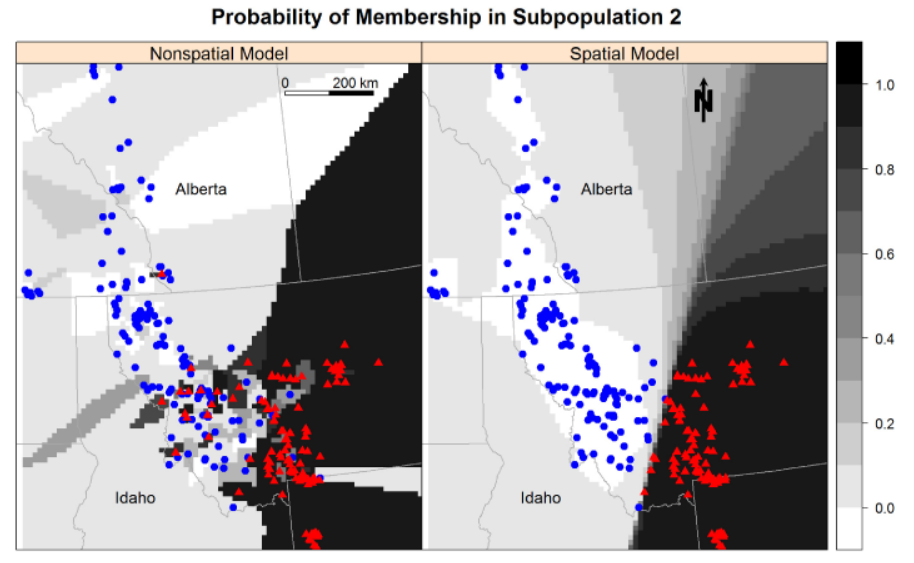

Figure 2. Posterior probabilities of belonging to subpopulation 2 from the non-spatial model and the spatial model.

of the sampled location and the random noise, $t_{i}=s_{i}+\delta_{i}$ (with an additive noise term $\delta_{i}$ sampled uniformly from a square with 25 mile sides and centered on $(0,0)$ ). This introduces a plausible representation of a home range for a typical cougar (Cougar Discussion Group 2009).

In both cases, the resulting subpopulation assignments from the correlated allele frequency model were exactly the same as with the uncorrelated allele frequency spatial model. The adjustment for cougar home ranges in the uncorrelated allele frequency spatial model changed the subpopulation assignment for three cougars along the area of division between the two subpopulations found earlier (results not shown). Three cougars in subpopulation 2 were switched to subpopulation 1 when adjusting for spatial uncertainty. This result is intuitive if the location of these cougars was moved from the east of a barrier to west of the barrier when adding spatial uncertainty, while the rest of the data still suggested the same location of the barrier. While the location of the barrier may be viewed as spatially fuzzier when adding spatial uncertainty to the sampling locations, the location of the barrier is determined endogenously from the data and will be determined from the majority of the data. The overall similarity in the composition of the subpopulations from the different models shows there is consistency in the results of the spatial Bayesian cluster models, even under different prior assumptions.

We next mapped the genetic subpopulations from the spatial model with the landscape features of interest. The overlap of genetic subpopulations and key landscape variables suggests that one or more landscape features may have influenced genetic structure in this cougar population (Fig. 3). Interstate Highway 15 aligns well with the subpopulations, as west of the highway is generally group 1 and east of the highway is group 2, where the interstate visually determines the membership for all but two cougars. This interstate highway has existed for decades, and was constructed 
along the route of another major road, US Route 91 . The Missouri River also separates the two subpopulations, for most cougars, in the northeast quadrant where the river is plotted on the map. There are six cougars from subpopulation 2 located on the west of the river and no cougars from subpopulation 1 located east of the river. Both the interstate highway and river are plausible physical barriers to cougar movement and gene flow. There is also a swath of low annual precipitation west of the Missouri River, east of Interstate 15 , which could also be a barrier to genetic flow. It is apparent that very few sampled cougars are located in areas with relatively low average annual precipitation. Average annual precipitation could be considered a direct measure of suit-

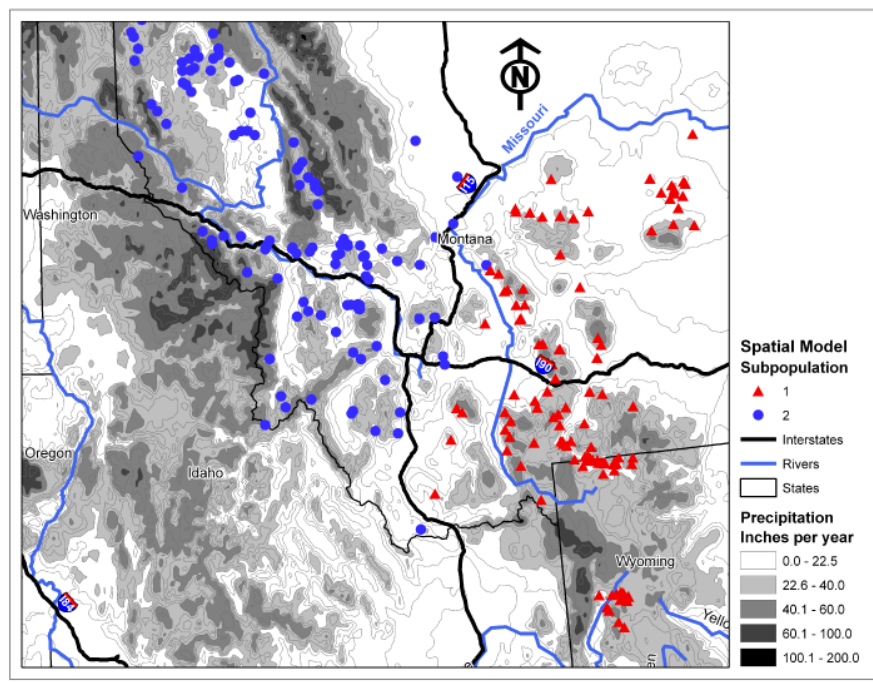

Figure 3. Posterior mode subpopulation membership assignments from the spatial model and major rivers, interstate highways, and average annual precipitation. able cougar habitat, as cougars favor forested areas, which require a certain minimum amount of precipitation. In summary, the boundary area separating genetic subpopulations of cougars corresponds to the spatial coincidence of three plausible landscape barriers: an area of relatively low annual precipitation, a major interstate highway, and a large river.

We next quantified the association between the landscape features and the genetic subpopulations using GLMs, specifically logistic regression since there are only two groups. Based on the map of genetic subpopulations and landscape features, we created an indicator variable for being located east of the Missouri River and an indicator variable for being east of the Interstate Highway 15. We first fitted models separately for each indicator variable to determine the relative importance of each landscape variable. A model with an intercept and the highway term was unstable and regression coefficients had large standard errors, so we compared models without intercepts. The Akaike information criterion (AIC) (Akaike, 1973) for the model with the Missouri River term was 232.52 and the AIC for the model with the I-15 term was 238.15 (Table 1). Both estimated regression coefficients were highly statistically significant and positively associated with the log-odds of being in subpopulation 2 : $\widehat{\beta}=4.74(p<0.001)$ for the major river and $\widehat{\beta}=3.69$ $(p<0.001)$ for the major highway. The AIC for a model with both landscape barrier variables was 232.42 , with regression coefficients $\widehat{\beta}=3.64(p=0.005)$ for the river and $\widehat{\beta}=1.10$ $(p=0.180)$ for the highway. Because the AIC was meaningfully lower for the river model than the highway model and only the river variable was statistically significant at the 0.05 level when both terms were included in the model, we excluded the highway term in subsequent models. We next fitted models with an intercept, river term, and with and without the mean annual precipitation variable. The AIC

Table 1. Estimated logistic regression model parameters (on linear predictor scale), standard errors, profile likelihood-based confidence intervals, p-values, and percent deviance explained and AIC for each model

\begin{tabular}{lccccccc}
\hline \hline Model & Beta & $\begin{array}{l}\text { Standard } \\
\text { Error }\end{array}$ & $\begin{array}{l}\text { Lower } \\
\text { Conf. Int. }\end{array}$ & $\begin{array}{l}\text { Upper } \\
\text { Conf. Int. }\end{array}$ & $p$-value & $\begin{array}{l}\text { Deviance } \\
\text { Explained }\end{array}$ & AIC \\
\hline $\begin{array}{l}\text { Model 1 } \\
\quad \text { Highway 15 }\end{array}$ & 3.689 & 0.585 & 2.716 & 5.090 & $<0.001$ & $36.90 \%$ & 238.15 \\
$\begin{array}{l}\text { Model 2 } \\
\quad \text { River }\end{array}$ & 4.736 & 1.004 & 3.240 & 7.605 & $<0.001$ & $38.40 \%$ & 232.52 \\
$\begin{array}{l}\text { Model 3 } \\
\quad \text { Highway 15 }\end{array}$ & 1.099 & 0.817 & -0.369 & 3.019 & 0.178 & $39.00 \%$ & 232.42 \\
$\quad$ River & 3.638 & 1.294 & 1.170 & 6.792 & 0.005 & & \\
Model 4 & & & & & & \\
$\quad$ Intercept & -3.232 & 0.416 & -4.167 & -2.506 & $<0.001$ & & 66.50 \\
$\quad$ River & 7.968 & 1.087 & 6.247 & 10.922 & $<0.001$ & & \\
Model 5 & & & & & & \\
$\quad$ Intercept & -3.937 & 0.845 & -5.864 & -2.517 & $<0.001$ & & \\
$\quad$ River & 7.926 & 1.090 & 6.199 & 10.883 & $<0.001$ & & \\
$\quad$ Precipitation & 0.031 & 0.029 & -0.025 & 0.088 & 0.280 & & \\
\hline \hline
\end{tabular}


Spatial Smooth in Model for Subpopulation 2 Status

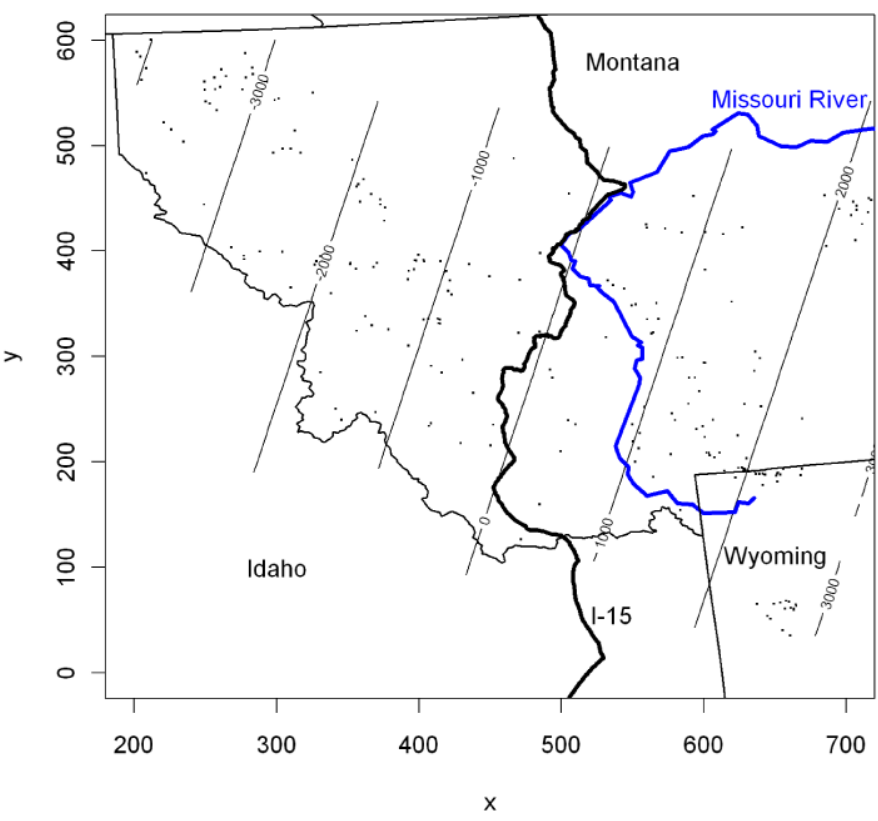

Figure 4. Bivariate smooth over geographic coordinates on the linear predictor scale (thin black contours) in a generalized additive model to explain Geneland subpopulation assignments, along with the Missouri River (starting in Wyoming and curving around to the northeast), Interstate highway l-15 (running roughly north-to-south), and sampled cougar locations.

was 66.50 for the model without the precipitation variable and 67.33 for the model with the precipitation term. The estimated regression coefficient for the river was $\widehat{\beta}=7.93$ $(p<0.001)$ and the coefficient for mean annual precipitation was $\widehat{\beta}=0.03(p=0.280)$. The results show that being located east of the Missouri River was significantly associated with an increased log-odds of being in subpopulation 2 , and mean annual precipitation had a positive association with the log-odds of being in this group, although not a significant one.

To construct a map of the modeled probability of belonging to subpopulation 2 and the Missouri River and Interstate Highway 15, we also fitted a GAM with an intercept and spatial smooth term and plotted the smoothed term and landscape features (Fig. 4). The contour line where the probability of belonging to subpopulation 2 is neutral (0 on linear predictor scale) lies primarily between the major river and highway, reinforcing the other model results that showed that both landscape features were associated with genetic population structure.

For validity of the subpopulation assignments, the highly significant $G$ test $(p<0.001)$ for genotypic differentiation in the Geneland clusters showed that the genotypes were not drawn from the same distribution for all subpopulations.
The $F_{S T}$ was 0.076 for the two subpopulations, indicating moderate gene flow between the two clusters. The standard error of $F_{S T}$ was 0.018 , for an approximate $95 \%$ confidence interval of $(0.039,0.112)$.

\section{DISCUSSION}

In this analysis, we found two spatially distinct genetic subpopulations of cougars using spatially explicit genetic models. These models allow a clearer picture of the geographic extent of each subpopulation than do the nonspatial models used previously by other researchers (Biek et al. 2006). We found that the genetic subpopulations spatially aligned with a confluence of landscape features, including a major highway, a major river, and low average annual precipitation. Our regression analyses confirmed the visual impression that there were several landscape barriers to genetic flow, and therefore, to movement and interaction of cougars. Our finding of genetic population structure that aligns with landscape features is consistent with findings from studies of cougars in other geographic areas (Ernest et al. 2003; McRae et al. 2005; Walker et al. 2000), and our study also provides evaluation of competing models and quantification of the impact of physical barriers that coincide with the detected boundary between genetic subpopulations. The landscape barriers of a major highway and a major river, and the low permeability area of low annual precipitation are all plausible barriers to cougar movement.

A natural question regarding our genetic population structure findings is how influential the spatial dependence prior is in the spatial model within Geneland. In other words, how confident should we be in our results of two spatially separated genetic subpopulations of cougars (in comparison to results from the non-spatial model)? While genetic population structure may be explained by the presence of certain limiting landscape features, there are other explanations for observed genetic structure, including Wright's $(1943 ; 1946)$ seminal hypothesis of isolation by distance (IBD), where a geographic pattern in genetic sequences is due to the expansion of the population and variation in sequences over time, i.e. a regular increase in population differentiation with increasing distance due to limited dispersal. The effect of a potential landscape barrier could be confounded by the presence of IBD. Overlapping spatial structure could also result from divergent populations with admixture (Falush et al. 2003) or from repopulation from two distinct subpopulations followed by population mixture in an area with historically suppressed population sizes, as argued by Biek et al. (2006) in the case of cougars.

Other researchers have performed simulation studies to assess the accuracy of the spatial model in Geneland, and we draw on their results. In a simulation study with datasets of varying levels of spatial organization, where the level of spatial organization was controlled by the number of Voronoi polygons (parameter $m$ ), Guillot et al. (2005a) found that 
the spatial model had lower false classification rates than the non-spatial model for highly $(m<12)$ and moderately $(m<25)$ spatially organized populations and performed as well as the non-spatial model when there was loose spatial organization $(m>80)$ in the simulated populations. Guillot et al. (2005a) also found through a simulation study that the spatial model did not force a spatial structure in genetic populations when it did not exist. Frantz et al. (2009) found in a simulation study that the spatial model in Geneland could correctly detect simulated barriers in data generated with true isolation by distance. Frantz et al. (2009) did find that the spatial model could overestimate the true number of subpopulations when a true pattern of strong IBD existed, but in our situation, with only two subpopulations and agreement in subpopulation number with the non-spatial model, this is not a concern. Manlove (2009) showed that the spatial model with correlated alleles had the overall lowest error rate in assignment of individuals to subpopulations when compared to the non-spatial model for simulated datasets with a range of population differentiation, as measured by the statistic $F_{S T}$. Given the previous simulation study findings and that the results from both the non-spatial model and the spatial model applied to the cougar data imply strong spatial organization, it is likely that in our case the spatial model is more accurate and captures latent spatial correlation in genotypes better than the non-spatial model.

Although our results are interesting and worthy of extending future research efforts, there are limitations to this study. One limitation is that while we find a correspondence in cougar genetic population structure and certain landscape features, we have not determined precisely which landscape barrier has affected genetic differentiation. There may be one influential landscape barrier or several coincident ones. We cannot formally infer covariate associations with the boundary within the existing Bayesian cluster model. Ideally, one would include relevant landscape covariates within the cluster model, such as suspected landscape barriers to animal movement and interaction, and quantify the impact of these landscape variables on the pattern of genetic differentiation. A challenge remains to separate the effects of several nearly coincident landscape features.

Another limitation is that we analyzed a sample of cougars that may not be representative of the cougar population in this area of North America. Most of the cougar samples were collected by hunters, and if there are areas with cougars where cougar hunting is restricted, the population will be likely underrepresented in these areas. This could potentially distort the pattern of genetic population structure we found if genetic population structure is related to landscape features in areas with no observations. Another drawback of this study is not having temporal data to correspond with the spatial data. Given the lack of sample time information available for each cougar, our spatial analysis does not capture variation in genetic structure over time.

While this paper focused on genetic population structure, there are implications of the work for disease ecology and landscape epidemiology. Disease ecology and landscape epidemiology involve studying the complex interactions in how a disease spreads over space, whether looking at the movement of the disease host or virus, often with the hope of curtailing or preventing future disease spread. Understanding genetic population structure of a species can lead to insight about animal movement and interaction and what role the landscape plays in the distribution of a host species. An improved understanding of the impact of landscape on host distribution and interaction could be used to more accurately predict the direction of disease spread in a population and allocate usually scarce resources accordingly. One important infectious disease in cougars is feline immunodeficiency virus (FIV), a fast-evolving virus found in several wild feline species (Biek et al. 2003). It may be reasonable to expect spatial pattern in the distribution of FIV in cougars, and also spatial structure in the feline immunodeficiency virus itself that is related to landscape due to the influence of landscape barriers. Previous work suggests that reduced genetic diversity was associated with reductions in disease immunity in several feline species, including cougars (Barone et al. 1994; Heeney et al. 1990; Wildt et al. 1987). Results of this study suggest that researchers should consider increasing usage of spatial information and spatial priors in Bayesian classification models when investigating genetic population structure to get a clearer picture of subpopulation definitions. By extension, results also suggest that one should consider the potential influence of landscape features on the spread of disease over space.

Our initial results are motivating, but there remain opportunities to continue and extend this line of research. One possibility is with a more formal evaluation of landscape effects in genetic population structure. One approach to the evaluation of landscape barriers in the role of species differentiation and disease transmission is an area of landscape ecology called wombling (Womble 1954), named for the first author to publish this idea. Approaches to wombling in the geography literature have been algorithmic (Monmonier 1979; Mu and Radke 2009), while approaches in the statistics literature have been model-based. Lu and Carlin (2005) first used a Bayesian hierarchical model-based framework for wombling and $\mathrm{Lu}$ et al. (2007) also considered covariates when performing Bayesian areal wombling. Wheeler and Waller (2008) used a Bayesian wombling model with spatially varying coefficients for particular landscape features to model disease spread over space. A similar model, with some modifications for multivariate genetic outcome data, could potentially be used with the cougar sample to estimate the influence of the discussed landscape features as barriers to gene flow. Accounting for spatial structure in genetic population structure appears important, and quantifying the landscape effects that influence spatial structure is necessary. This situation provides opportunity for further methodological research developments. 


\section{ACKNOWLEDGEMENTS}

We thank Dr. Roman Biek for providing access to the cougar data and for helpful comments on an earlier version of this manuscript.

\section{Received 28 February 2011}

\section{REFERENCES}

AKaike, H. (1973). Information theory and an extension of the maximum likelihood principle, in International Symposium on Information Theory, B. Petran and F. Csaaki, eds. Budapest, Hungary. pp. 267-281. MR0483125

Allendorf, F. W. and Luikart, G. (2006). Conservation and the Genetics of Popualtions. Wiley-Blackwell, Hoboken, New Jersey.

Anderson, C. R., Lindzey, F. G., and McDonald, D. B. (2004). Genetic structure of cougar populations across the Wyoming Basin: Metapopulation or megapopulation? Journal of Mammology $\mathbf{8 5}$ $1207-1214$.

Avise, J. C. (2000). Phylogeography: The History and Formation of Species. Harvard University Press, Cambridge, Massachusetts.

Barone, M. A., Roelke, M. E., Howard J., Brown, J. L., AnDERSON, A. E., and Wildt, D. E. (1994). Reproductive characteristics of male Florida panthers: Comparative studies from Florida, Texas, Colorado, Latin America, and North American zoos. Journal of Mammalogy $\mathbf{7 5}$ 150-162.

Berk, R. (2008). Statistical Learning from a Regression Perspective. Springer, New York. MR2722293

Biek, R., Drummond, A., and Poss, M. (2006). A virus reveals population structure and recent demographic history of its carnivore host. Science 311 538-541.

Biek, R., Rodrigo, A. G., Holley, D., Drummond, A., Anderson, C. R., Ross, H. A., and Poss, M. (2003). Epidemiology, genetic diversity, and evolution of endemic feline immunodeficiency virus in a population of wild cougars. Journal of Virology 77 9578-9589.

Castric, V., Bonney, F., and Bernatchez, L. (2001). Landscape structure and hierarchical genetic diversity in the brook charr, Salvelinus fontinalus. Evolution 55 1016-1028.

Cegelski, C., Waits, L. P., and Anderson, N. J. (2003). Assessing population structure and gene flow in Montana wolverines (Gulo gulo) using assignment-based approaches. Molecular Ecology 12 2907-2918.

Conner, J. K. and Harte, D. L. (2004). A Primer of Ecological Genetics. Sinauer Associates, Sunderland, MA.

Cougar Discussion Group (2009). Utah Cougar Management Plan v. 2.0. Utah Division of Wildlife Resources. http://wildlife. utah.gov/pdf/cmgtplan.pdf. Accessed 30 September 2010.

Coulon, A., Gulllot, G., Cosson, J., Angibault, J. M. A., Aulagnier, S., Cargnelutti, B., Galan, M., and Hewison, A. J. M. (2006). Genetics structure is influenced by landscape features: Empirical evidence from a roe deer population. Molecular Ecology 15 1669-1679.

EpPerson, B. K. (2003). Geographical Genetics. Princeton University Press, Princeton.

Epps, C. W., Palsboll, P. J., Wehausen, J. D., Roderick, G. K., Ramey, R. R., and McCullough, D. R. (2005). Highways block gene flow and cause a rapid decline in genetic diversity of desert bighorn sheep. Ecology Letters 8 1029-1038.

Ernest, H. B., Boyce, W. M., Bleich, V. C., May, B., Stiver, S. J., and Torres, S. G. (2003). Genetic structure of mountain lion (Puma concolor) populations in California. Conservation Genetics 4 353-366.

ESRI (2005). ArcGIS 9.1 Users Guide. Environmental Systems Research Institute, Redlands, California.

Falush, D., Stephens, M., and Pritchard, J. K. (2003). Inference of population structure using multilocus genotype data: Linked loci and correlated allele frequencies. Genetics 164 1567-1587.
Frankham, R., Ballou, J. D., and Briscoe, D. A. (2010). Introduction to Conservation Genetics, 2nd ed. Cambridge University Press, Cambridge.

Frantz, A. C., Cellina, S., Krier, A., Schley, L., and Burke, T. (2009). Using spatial Bayesian methods to determine the genetic structure of a continuously distributed population: Clusters or isolation by distance? Journal of Applied Ecology 46 493-505.

Frantz, A. C., Tigel Pourtois, J., Heuertz, M., Schley, L., Flamand, M. C., Krier, A., Bertoullle, S., Chaumont, F., and Burke, T. (2006). Genetic structure and assignment tests demonstrate illegal translocation of red deer (Cervus elaphus) into a continuous population. Molecular Ecology 15 3191-3203.

Freeland, J. (2006). Molecular Ecology. John Wiley and Sons, West Sussex.

Funk, W. C., Blouin, M. S., Corn, P. S., Maxell, B. A., Pilliod, D. S., Amish, S., and Allendorf, F. W. (2005). Population structure of Columbia spotted frogs (Rana luteiventris) is strongly affected by the landscape. Molecular Ecology 14 483-496.

Goudet, J. (2005). HIERFSTAT, a package for R to compute and test hierarchical F-statistics. Molecular Ecology Notes 5 184-186.

Goudet, J., Raymond, M., DeMeeus, T., and Rousset, F. (1996). Testing differentiation in diploid populations. Genetics 144 19331940.

Guillot, G. (2008). Inference of structure in subdivided populations at low levels of genetic differentiation-The correlated allele frequencies model revisited. Bioinformatics 24 2222-2228.

Guillot, G., Estroup, A., Motrier, F., and Cosson, J. F. (2005a). A spatial statistical model for landscape genetics. Genetics $\mathbf{1 7 0}$ $1261-1280$.

Guillot, G., Leblois, R., Coulon, A., and Frantz, A. C. (2009). Statistical methods in spatial genetics. Molecular Ecology 18 47344756 .

Guillot, G., Motrier, F., and Estoup, A. (2005b). Geneland: A computer package for landscape genetics. Molecular Ecology Notes 5 712-715.

Guillot, G., Santos, F., and Estoup, A. (2008). Analysing georeferenced population genetics data with Geneland: A new algorithm to deal with null alleles and a friendly graphical user interface. Bioinformatics 24 1406-1407.

Guillot, G., Santos, F., and Estoup, A. (2011). Population genetics analysis using $\mathrm{R}$ and Geneland. http://www2.imm.dtu.dk/ rigu/Geneland/Geneland-Doc.pdf. Accessed 14 June 2011.

Guo, S. W., and Thompson, E. A. (1992). Performing the exact tests of Hardy-Weinberg proportion for multiple alleles. Biometrics $\mathbf{4 8}$ $361-372$

HARDY, O., and VeKemans, X. (2002). SPAGeDi: A versatile computer program to analyse spatial genetic structure at the individual or population levels. Molecular Ecology Notes 2 618-620.

Hartu, D. L. (2000). A Primer of Population Genetics, 3rd ed. Sinauer Associates, Sunderland, MA.

Hastie, T., Tibshirani, R., and Friedman, J. (2001). The Elements of Statistical Learning: Data Mining, Inference, and Prediction. Springer, New York. MR1851606

HeDrick, P. W. (2004). Genetics of Populations, 3rd ed. Jones \& Bartlett Learning, Sudbury, MA.

Heeney, J. L., Evermann, J. F., McKeirnan, A. J., Markerkraus, L., Roelke, M. E., Bush, M., Wildt, D. E., Meltzer, D. G., Colly, L., Lukas, J., Manton, V. J., Caro, T., and O'Brien, S. J. (1990). Prevalence and implications of feline coronavirus infections of captive and free-ranging cheetahs (Acinonyx jubatus). Journal of Virology 64 1964-1972.

Holderegger, R., Wagner, H. H. (2008). Landscape genetics. BioScience 58 199-207.

Keyghobadi, N., Roland, J., and Strobeck, C. (1999). Influence of landscape on the population structure of the alpine butterfly Parnassius smintheus (Papilionidae). Molecular Ecology 8 1481-1495.

Loxterman, J. L. (2001). The Impact of Habitat Fragmentation on the Population Genetic Structure of Pumas (Puma concolor) in Idaho. Dissertation, Idaho State University. 
Lu, H., and Carlin, B. P. (2005). Bayesian areal wombling for geographical boundary analysis. Geographical Analysis 37 265-285.

Lu, H., Reilly, C. S., Baneruee, S., and Carlin, B. P. (2007). Bayesian areal wombling via adjacency modeling. Environmental and Ecological Statistics 14 433-452. MR2405556

Manel, S., Schwartz, M., Luikart, G., and Taberlet, P. (2003). Landscape genetics: Combining landscape ecology and population genetics. Trends in Ecology and Evolution 18 189-197.

Manlove, K. (2009). Algorithm Selection for Models in Landscape Genetics: A Statistician's Guide. Unpublished MS Thesis, Montana State University.

McCairns, R. J. S., and Bernatchez, L. (2008). Landscape genetic analyses reveal cryptic population structure and putative selection gradients in a large-scale estuarine environment. Molecular Ecology 17 3901-3916.

McRae, B. H., Beier, P., Dewald, L. E., Huynh, L. Y., and Keim, P. (2005). Habitat barriers limit gene flow and illuminate historical events in a wide-ranging carnivore, the American puma. Molecular Ecology 14 1965-1977.

Monmonier, M. S. (1973). Maximum-difference barriers: An alternative numerical regionalization method. Geographic Analysis 5 245261.

Mu, L., and RAdKe, J. (2009). A weighted difference barrier method in landscape genetics. Journal of Geographical Systems 11 141-154.

Piertney, S., MacColl, A. D. C., Bacon, P. J., and Dallas, J. F. (1998). Local genetic structure in red grouse (Lagopus lagopus scoticus): Evidence from microsatellite DNA markers. Molecular Ecology 7 1645-1654.

Pritchard, J., Stephens, M., and Donnelly, P. (2000). Inference of population structure using multilocus genotype data. Genetics $\mathbf{1 5 5}$ 945-959.

RAymond, M., and Rousset, F. (1995a). GENEPOP (version 1.2): Population genetics software for exact tests and ecumenicism. Journal of Heredity 86 248-249.

Raymond, M., and Rousset, F. (1995b). An exact test for population differentiation. Evolution 49 1283-1286.

ReAl, L. A., and Biek, R. (2007). Spatial dynamics and genetics of infectious diseases on heterogeneous landscapes. Journal of the Royal Society Interface 4 935-948.

Riley, S. P. D., Pollinger, J. P., Sauvajot, R. M., York, E. C., Bromley, C., Fuller, T. K., and Wayne, R. K. (2006). A southern California freeway is a physical and social barrier to gene flow in carnivores. Molecular Ecology 151733-1741.

Rousset, F. (2004). Genetic Structure and Selection in Subdivided Populations. Princeton University Press, Princeton.

Sacks, B. N., Bannasch, D. L., Chomel, B. B., and Ernest, H. B. (2008). Coyotes demonstrate how habitat specialization by individuals of a generalist species can diversify populations in a heterogeneous ecoregion. Molecular Biology and Evolution 25 1354-1395.

Sacks, B. N., Brown, S. K., and Ernest, H. B. (2004). Population structure of California coyotes corresponds to habitat-specific breaks and illuminates species history. Molecular Ecology 13 1265-1275.

Sinclair, E. A., Swenson, E. L., Wolfe, M. L., Choate, D. C., Bates, B., and Crandall, K. A. (2001). Gene flow estimates in
Utah's cougars imply management beyond Utah. Animal Conservation 4 257-264.

Storfer, A., Murphy, M. A., Evans, J. S., Goldberg, C. S., Robinson, S., Spear, S. F., Dezzani, R., Demelle, E., Vierling, L., and Waits, L. P. (2007). Putting the 'landscape' in landscape genetics. Heredity 98 128-142.

Storfer, A., Murphy, M. A., Spear, S. F., Holderegger, R., and Waits, L. P. (2010). Landscape genetics: Where are we now? Molecular Ecology 19 3496-3514.

Walker, C. W., Harveson, L. A., Pittman, M. T., Tewes, M. E., and Honeycutt, R. L. (2000). Microsatellite variation in two populations of mountain lions (Puma concolor) in Texas. Southwestern Naturalist 45 196-203.

Weir, B. S. and Cockerham, C. C. (1984). Estimating F-statistics for the analysis of population structure. Evolution 38 1358-1370.

Wheeler, D. C. and Waller, L. A. (2008). Mountains, rivers, and valleys: The transmission of raccoon rabies over a heterogeneous landscape. Journal of Agricultural, Biological, and Environmental Statistics 13 388-406. MR2590936

Wildt, D. E., Bush, K. L., Goodrowe, K. L., Packer, C., Pusey, A. E., Brown, J. L., Joslin, P., and O'Brien, S. J. (1987). Reproductive and genetic consequences of founding isolated lion populations. Nature 329 328-331.

Womble, W. H. (1951). Differential systematics. Science 114 315-322.

Wood, S. (2006). Generalized Additive Models: An Introduction with $R$. Chapman \& Hall/CRC, Boca Raton. MR2206355

Wright, S. (1943). Isolation by distance. Genetics 28 114-138.

Wright, S. (1946). Isolation by distance under diverse systems of mating. Genetics 31 39-59.

David C. Wheeler

National Cancer Institute

6120 Executive Blvd

MSC 7240, EPS 8109

Rockville, MD 20852

USA

Tel.: $301-435-4702$

Fax: 301-402-1819

E-mail address: dcwheels@gmail.com

Lance A. Waller

Emory University Department of Biostatistics and Bioinformatics

1518 Clifton Rd., N.E., 3rd Floor

Rollins School of Public Health

Atlanta, GA 30322

USA

E-mail address: Iwaller@emory.edu 